\title{
Diagnostic value of hematological parameters in acute pancreatitis
}

\author{
Liang Wang ${ }^{1,2 \#}$, Xinwei Qi ${ }^{1,2 \#}$, Fengming Tian ${ }^{1}$, Huijun $\mathrm{Li}^{1,3}$, Hui Zhao ${ }^{1,2}, \mathrm{Jie} \mathrm{Lv}^{1,2}$, Xuan Zhou ${ }^{1,2}$, \\ Derong $\mathrm{Ba}^{1,2}$, Wen Song ${ }^{1,2}$, Xiumin $\mathrm{Ma}^{1,2,4}$
}

${ }^{1}$ State Key Laboratory of Pathogenesis, Prevention and Treatment of High Incidence Diseases in Central Asia, Clinical Laboratory Center, Tumor Hospital Affiliated to Xinjiang Medical University, Urumqi, China; ${ }^{2}$ First Affiliated Hospital of Xinjiang Medical University, Urumqi, China; ${ }^{3}$ Department of Blood Transfusion, People's Hospital of Xinjiang Uygur Autonomous Region, Urumqi, China; ${ }^{4}$ College of Basic Medicine of Xinjiang Medical University, Urumqi, China

Contributions: (I) Conception and design: X Qi, H Li; (II) Administrative support: L Wang, X Ma; (III) Provision of study materials or patients: F Tian, H Zhao; (IV) Collection and assembly of data: J Lv, X Zhou; (V) Data analysis and interpretation: D Ba, W Song; (VI) Manuscript writing: All authors; (VII) Final approval of manuscript: All authors.

\#These authors contributed equally to the work.

Correspondence to: Xiumin Ma. State Key Laboratory of Pathogenesis, Prevention and Treatment of High Incidence Diseases in Central Asia, Clinical Laboratory Center, Tumor Hospital Affiliated to Xinjiang Medical University, First Affiliated Hospital of Xinjiang Medical University, College of Basic Medicine of Xinjiang Medical University, No. 789 Suzhou Road, Urumqi 830011, China. Email: maxiumin1210@sohu.com.

Background: Acute pancreatitis (AP) is an inflammatory disease with rapid onset and rapid progression. In severe cases, it can cause systemic inflammatory response syndrome (SIRS), multiple organ failure (POF) and death. In order to explore the effective index of early diagnosis of AP, we have detected the levels of serum lipase (LPS), serum amylase (S-AMY) and C-reactive protein (CRP). Meanwhile, cost evaluation was performed on these biochemical parameters for AP patients.

Methods: In this study, 212 patients were admitted with acute abdominal pain, divided into AP group $(\mathrm{n}=107)$ and non-AP group $(\mathrm{n}=105)$, including cholelithiasis $(\mathrm{n}=8)$, intestinal obstruction $(\mathrm{n}=34)$, acute cholangitis $(n=14)$, acute cholecystitis $(n=20)$, acute appendicitis $(n=20)$ and pancreatic cancer $(n=9)$. Demographic data and laboratory examination results were collected, the sensitivity and specificity of LPS, $\mathrm{S}-\mathrm{AMY}$ and CRP in the diagnosis of AP were calculated respectively, as well as the costs of single or multiple tests were evaluated.

Results: The serum level of S-AMY and LPS in AP group were significantly higher than those in nonAP group $(\mathrm{P}<0.01)$, The serum level of $\mathrm{CRP}$ in AP group was higher than that in non-AP group $(\mathrm{P}<0.05)$. When the critical value of the normal reference value is three times as the critical value, the sensitivity and specificity of the LPS in diagnosing AP were $91.6 \%$ and $90.5 \%$, S-AMY was $70.1 \%$ and $91.4 \%$. And CRP was $59.8 \%$ and $56.2 \%$, respectively. Compared with the trend of serum LPS expression, the expression trend of S-AMY was positively correlated $(r=0.81)$, while there was no correlation with the trend of CRP expression.

Conclusions: The ability of LPS to diagnose AP is significantly better than that of LPS + CRP, as well as the combined detection of LPS + CRP + S-AMY, which can be used as the first choice for laboratory examination of AP.

Keywords: Acute pancreatitis (AP); lipase; serum amylase (S-AMY); C-reactive protein (CRP)

Submitted Jan 09, 2020. Accepted for publication Jun 15, 2020.

doi: $10.21037 /$ apm-20-160

View this article at: http://dx.doi.org/10.21037/apm-20-160 


\section{Introduction}

Acute pancreatitis (AP) is one of the most common diseases with acute abdominal pain. It is related to diverse causes with complicated pathogenesis and high mortality. When pancreatic enzyme is activated, pancreatic acinars are damaged and a large amount of pancreatic enzymes released to cause pancreatic tissues into self-digest, edema, and bleeding, then leading to local inflammatory reactions (1). Acute inflammatory reaction syndrome can occur in some severe cases, AP is sometimes fatal, with death occurring in $3 \%$ of cases (2), which has brought tremendous suffering to patients as well as huge difficulties in clinical diagnosis and treatment (3). AP needed to be diagnosed early so that therapeutic interventions can be carried out in time. Normally, AP is diagnosed based on elevated levels of pancreatic enzymes in the blood or urine, accompanied by abdominal pain and imaging findings (4).

Currently, Diagnosis of AP was based on the presence of at least two of the following three criteria: (I) acute onset of a persistent, severe epigastric pain often radiating to the back. (II) Serum lipase (LPS) activity (or amylase activity) at least three times greater than the upper limit of normal (ULN). (III) Characteristic findings of AP on contrast enhanced computed tomography (CECT) and, less commonly, magnetic resonance imaging (MRI) or transabdominal ultrasonography (5). Combined serum amylase (S-AMY) with LPS detection is frequently used to diagnose AP due to their effectiveness, which is uncorrelated with pancreatitis severity. Research in recent years has pointed out that LPS is an indicator with a higher sensitivity and specificity than S-AMY, which can be used alone to detect AP (6).

C-reactive protein $(\mathrm{CRP})$ is an acute phase reaction protein, which has a little content in a healthy human. However, its content will rapidly increase in the case of infection and inflammation (7). When the body's inflammation or damage is reduced, its concentration also drops accordingly. And long-term high CRP level generally indicates poor prognosis for patients (8).

Nowadays, the laboratory examination of AP is mostly combined detection of serum LPS and S-AMY. This research was thereby carried out aiming to compare the sensitivity and specificity of serum LPS, S-AMY and CRP in the diagnosis of AP. Then evaluating the value of these indicators in clinical diagnosis of AP. Meanwhile, detection expense for patients admitted with acute abdominal pain were also evaluated. Above all, according to the laboratory hematology examination, we aimed to investigate whether hematological parameters LPS, S-AMY and CRP have an important initial clear diagnosis in the differential diagnosis. The initial diagnosis of AP gives clinicians a clear diagnosis and treatment direction, and active treatment is of great significance in improving the patients' prognosis, reducing economic pressure, saving medical resources, and so on.

\section{Methods}

\section{General information}

In the study, there were 212 patients with acute abdominal pain admitted to the hospital from 2014 to 2016, 143 males/69 females, the ages between 17-87 years old. According to the guidelines for the diagnosis of AP (5), we combined with clinical symptoms of patients: abdominal pain and vomiting, laboratory tests: serum trypsin (S-AMY or LPS) increased three times higher than normal, and CT results to diagnose AP. AP was divided into the following categories: (I) gallstone pancreatitis: founding gallbladder stones or intrahepatic bile duct thickening, enhancement, biliary ascariasis through imaging tests. (II) Alcoholic pancreatitis: drinking caused AP or has a history of alcoholism (male: the amount of alcohol $>40 \mathrm{~g} /$ day, female: the amount of alcohol $>20 \mathrm{~g} /$ day, and wine storage time $\geq 5$ years or the average drinking accuracy in the last 2 weeks was $80 \mathrm{~g} /$ days), it has the lack of other obvious evidence of the disease (5). (III) Hyperlipidemia pancreatitis: the level of serum triglyceride $>11.3 \mathrm{mmol} / \mathrm{L}$, or the serum is opaque, the level of serum triglyceride is between 5.65 and $11 \mathrm{mmol} / \mathrm{L}$, with no any other pathogenic factor obviously (9). (IV) Idiopathic pancreatitis: with no determined factors after clinical, imaging studies, biochemistry, etc. (10). One hundred and seven patients were diagnosed with AP, with 78 males/29 females, ages between 21-86 years old, the patients average age is $49.2 \pm 16.85$ years old; 105 patients were diagnosed with non$\mathrm{AP}$, containing 65 males and 40 females, ages between 17 87 years old, the patients average age was $47.6 \pm 15.02$ years old. The data collected was comparable, there was no significant difference in $\mathrm{AP}$ group and non-AP group $(\mathrm{P}>0.05)$. All the laboratory tests were completed within 12 hours after hospitalized. Meanwhile, the cases more than $24 \mathrm{~h}$ on the admission day were taken out. Our study was conducted in accordance with the Declaration of Helsinki (as revised in 2013). The study protocols were approved by the ethics committee of the First Affiliated Hospital of Xinjiang 
Table 1 The serum levels comparison of LPS, S-AMY and CRP

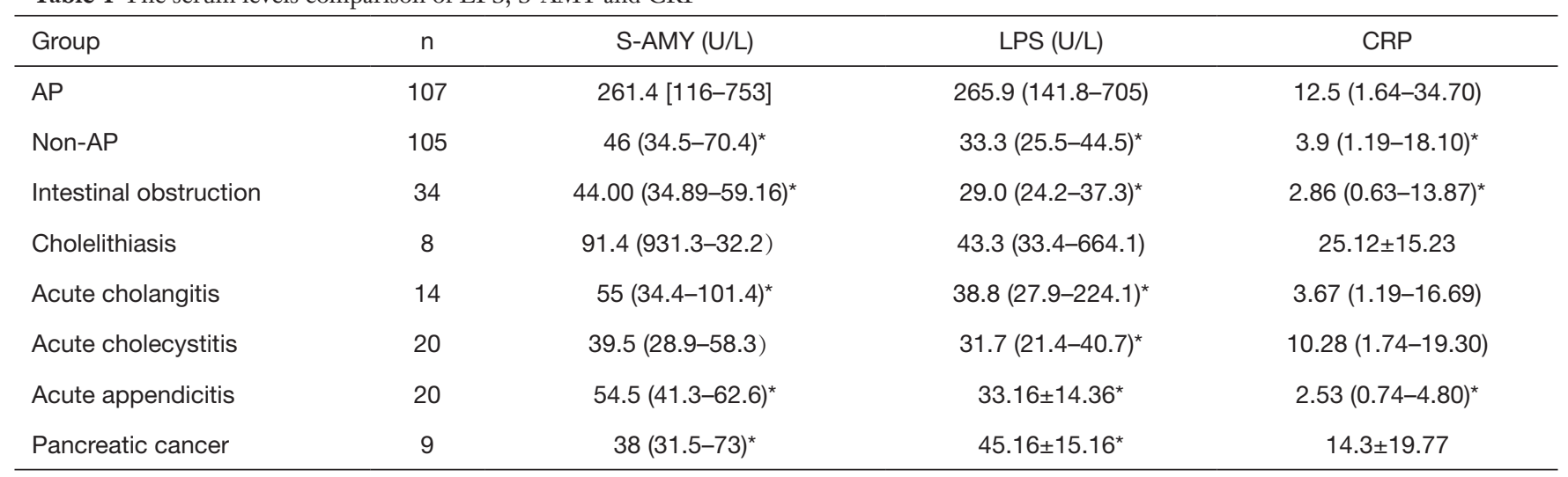

*, $\mathrm{P}<0.01$, compared with the healthy control group. LPS, serum lipase; S-AMY, serum amylase; CRP, C-reactive protein.

Medical University (approval No. 20140828-02) and received informed consent from all subjects.

\section{Instrument and reagent}

S-AMY, LPS (Beckman original reagent, USA), and CRP (Jiuqiang Biotechnology Company, Beijing, China) were determined by an automatic blood biochemical analyzer (Beckman Counter DXC800, USA).

\section{Test methods}

All patients were collected blood $6 \mathrm{ml}$ in 6 hours after admitted, methods detected the serum level of LPS and S-AMY by using velocity assays, methods detected the serum level of CRP using atex particle immunoturbidimetry. All operations were strictly in accordance with the instructions.

\section{Cost evaluation}

Separating detection of the levels of pancreatic enzyme (S-AMY or LPS) cost $15 \mathrm{RMB}$, and the detection of AP (S-AMY and LPS) cost $30 \mathrm{RMB}$ and the CRP test cost 20 $\mathrm{RMB}$ respectively.

\section{Statistical analysis}

All data analysis was using SPSS 22.0. The non-parametric data was expressed by median, parametric data was expressed by mean \pm standard deviation (SD). Variance analysis was used for two groups' differences. Calculating the sensitivity and specificity of LPS, S-AMY, CRP respectively, and make a comparison of them. $\mathrm{P}<0.05$ indicated statistical significance.

\section{Results}

\section{The serum levels of LPS, S-AMY and CRP}

There were 107 patients in the AP group and 105 patients in the non-AP group. The levels of LPS, S-AMY, CRP in $\mathrm{AP}$ group were higher than that in non-AP group, and it was significantly different between two groups. The serum levels of LPS, S-AMY in AP group was obviously higher than in non-AP group $(\mathrm{P}<0.01)$. The serum level of $C R P$ in $\mathrm{AP}$ group was also obviously higher than in non-AP group $(\mathrm{P}<0.05)$ (shown in Table 1).

\section{The sensitivity and specificity of LPS, S-AMY, CRP in AP group}

The sensitivity and specificity of the LPS in diagnosing AP were $91.6 \%$ and $90.5 \%$, S-AMY was $70.1 \%$ and $91.4 \%$. CRP was $59.8 \%$ and $56.2 \%$, respectively. To compare the sensitivity and specificity of LPS, S-AMY, CRP in the early diagnose of AP group, we discovered the sensitivity of LPS, which was obviously higher than S-AMY and CRP $(\mathrm{P}<0.01)$, the specificity of $\mathrm{S}-\mathrm{AMY}$ was slightly higher than LPS, there was no significant difference between them $(\mathrm{P}>0.05)$ (details shown in Table 2$)$. In addition, compared with the trend of serum LPS expression, the expression trend of S-AMY was positively correlated $(\mathrm{r}=0.81)$ (Figure 1). While there was no correlation with the trend of CRP expression (Figure 2).

In AP group, there were no patients with S-AMY 
Table 2 The sensitivity and specificity of LPS, S-AMY, CRP in AP group

\begin{tabular}{lll}
\hline Test index & Sensitivity & Specificity \\
\hline S-AMY & $70.1^{* \star}$ & 91.4 \\
LPS & 91.6 & 90.5 \\
CRP & $59.8^{\star \star}$ & $56.2^{\star *}$ \\
S-AMY + LPS & $64.2^{\star *}$ & 99.1 \\
LPS + CRP & $54.7^{\star *}$ & 95 \\
S-AMY + CRP & $41.9^{\star *}$ & 96.1 \\
S-AMY + LPS + CRP & $41.9^{\star *}$ & 96.1 \\
\hline
\end{tabular}

**, $\mathrm{P}<0.01$, compared with serum lipase. LPS, serum lipase; $\mathrm{S}-\mathrm{AMY}$, serum amylase; CRP, C-reactive protein; AP, acute pancreatitis.

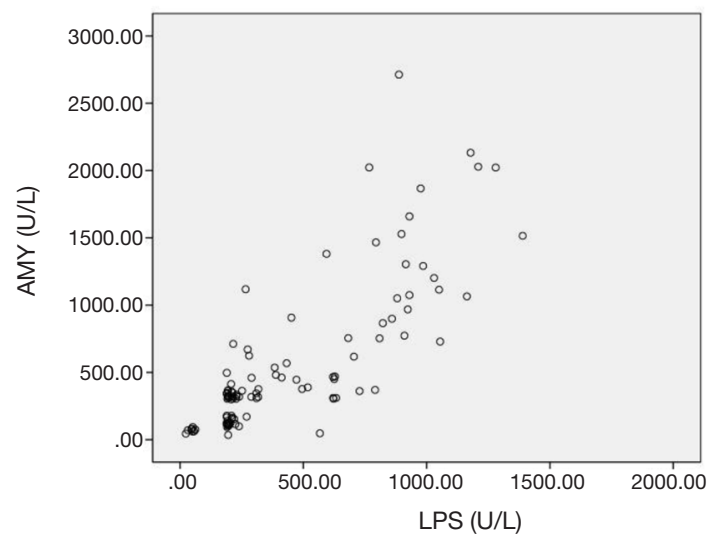

Figure 1 Correlation analysis between serum level of LPS and S-AMY. LPS, serum lipase; S-AMY, serum amylase.

elevated but normal LPS. The proportion of LPS rise of patients who had biliary pancreatitis, hyperlipidemic pancreatitis and alcoholic pancreatitis were 10.6\%, 11.5\% and $40 \%$ respectively. The patients with increased serum levels of S-AMY and LPS accounted for $77.57 \%$. The patients with normal serum levels of S-AMY and LPS accounted for $7.5 \%$ of the total number (details shown in Table 3).

\section{Cost evaluation}

It cost 50 RMB when test was combined in LPS, S-AMY and CRP, test of LPS individually cost only 15 RMB. Two hundred and twelve patients with acute abdomen could be tested LPS individually which could save 7,420 RMB in

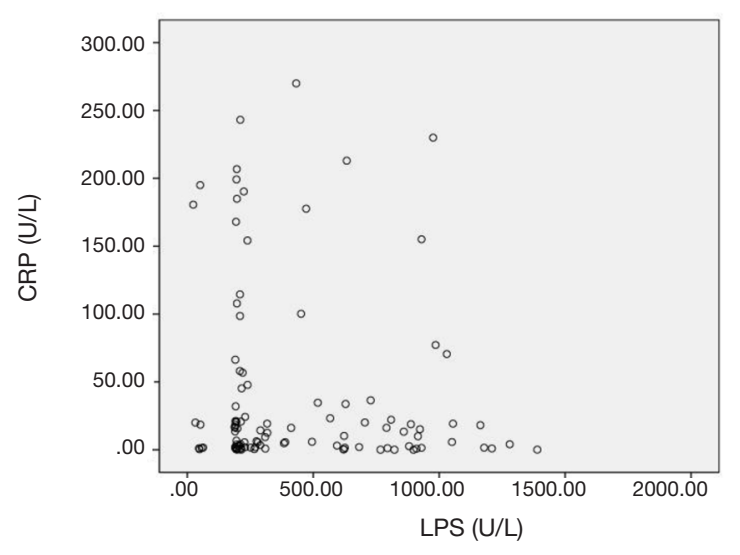

Figure 2 Correlation analysis between serum level of LPS and CRP. LPS, serum lipase; CRP, C-reactive protein.

our study.

\section{Discussion}

Early clinical diagnosis is the most important in treatment of acute abdomen patients. However, research discovered that the accurate diagnosis rate of acute abdomen patients is only $47-76 \%$, while delayed diagnosis will increase the morbidity and mortality in patients (11). AP is one of the common acute abdomens, which manifests as persistent upper abdominal pain, accompanying with discomforts like abdominal distention, nausea and vomiting. Mild cases are dominated by local pancreatic inflammatory reaction, while severe cases may be accompanying with organ dysfunction. Therefore, it's crucial importance to clinical treatment to search for a suitable detection index that can timely and accurately diagnose AP.

At present, S-AMY as the detection index most commonly is used in diagnosing AP. S-AMY can secrete specific subtype via salivary gland (s-amylase) and pancreas, which has the main function of digesting starch, glycogen and related polysaccharide (12). S-AMY has extensive sources, which can be elevated in acute abdomens such as parotitis, cholecystitis and peptic ulcer. Therefore, the specificity of S-AMY in diagnosing AP is not high (13). S-AMY rises rapidly in 3-6 h after onset of AP, with a half-life of $12 \mathrm{~h}$. It can return to normal concentration within $24 \mathrm{~h}$, which has greatly reduced value as a clinical early diagnosis index (12). LPS is synthesized in pancreatic acinar cells, which plays a vital role in lipolysis. LPS concentration in pancreas is 100 -fold higher than that in tissues such as duodenum, stomach and lung. Generally, LPS will increase 3-6 h after onset of AP, 
Table 3 The levels of LPS and S-AMY in AP group with different etiology

\begin{tabular}{|c|c|c|c|c|c|c|}
\hline Type & $\mathrm{n}$ & LPS $\rightarrow$ AMY $\rightarrow(n, \%)$ & LPS $\uparrow A M Y \uparrow(n, \%)$ & LPS $\uparrow A M Y \rightarrow(n, \%)$ & LPS $\rightarrow$ AMY $\uparrow(n, \%)$ & $>3$ times LPS $(n, \%)$ \\
\hline Hyperlipidemic & 26 & 0 & $23(88.5)$ & $3(11.5)$ & 0 & $26(100.0)$ \\
\hline Alcoholic & 20 & $2(10.0)$ & $10(50.0)$ & $8(40.0)$ & 0 & $17(85.0)$ \\
\hline Idiopathic & 14 & $1(7.1)$ & $13(92.9)$ & 0 & 0 & $13(92.9)$ \\
\hline
\end{tabular}

LPS $\rightarrow$, normal level of serum lipase; AMY $\rightarrow$, normal level of serum amylase; LPS $\uparrow$, increased level of serum lipase; AMY $\uparrow$, increased level of serum amylase.

LPS as the preferred serological test for the diagnosis of pancreatitis and a cut-off level of three or more times the ULN is often quoted. which peaks at $24 \mathrm{~h}(12,14)$. Different from S-AMY, LPS can be reabsorbed by renal tubule. Consequently, elevation in LPS concentration can last for 8-14 days, indicating greater value of LPS than S-AMY in diagnosing AP when the duration of disease is more than $24 \mathrm{~h}$ (12). It was found in this research that, the level of S-AMY and LPS in AP patients was higher than that in non-AP patients with the difference being statistically significant $(\mathrm{P}<0.01)$.

Recently, numerous studies indicate that LPS displays higher sensitivity and specificity than S-AMY in diagnosing AP. It is pointed out in American College of Gastroenterology (ACG, 2013) and "JPN guideline for the management of Acute Pancreatitis" published by Japan Association of Hepatobiliary and Pancreatic Surgery that LPS shows higher specificity in diagnosing AP compared with S-AMY. Therefore, LPS is recommended in the two guidelines as the preferred diagnostic index (3). The research of AP (15) indicated that LPS had the sensitivity and specificity of $86.5-100 \%$ and $84.7-99.0 \%$ in diagnosing AP respectively. The research of Dhanwant Gomez et al. (6) suggested that the sensitivity and specificity of LPS were $96.6 \%$ and $99.4 \%$ respectively in diagnosing AP, while those of S-AMY were $78.6 \%$ and $99.1 \%$ respectively. Thus, it can be seen that LPS shows higher sensitivity and specificity than S-AMY. Furthermore, determination of LPS level alone is sufficient enough to confirm AP, which can greatly save the detection expenses (6,16-18). Batra et al. (13) also drew a similar conclusion in their research. Furthermore, the serum level of S-AMY and LPS in $84 \%$ AP patients both increased, while the serum level of LPS increased in $100 \%$ AP patients under undifferentiated etiology condition. The proportions of only increased LPS level in biliary pancreatitis, hyperlipidemic pancreatitis, and alcoholic pancreatitis patients were $10.6 \%, 11.5 \%$ and $40 \%$ respectively. In this study, the sensitivity and specificity of LPS and S-AMY in diagnosing AP were similar to previous studies. Specifically, the sensitivity and specificity of only increased LPS level in diagnosing $\mathrm{AP}$ were $91.6 \%$ and $90.5 \%$ respectively, while those of only increased S-AMY level were $70.1 \%$ and $91.4 \%$. Moreover, the specificity of combining S-AMY with LPS level in diagnosing AP was 99.1\%, which was higher than that of only detecting LPS level. However, the difference was not statistically significant $(P>0.05)$. It indicated that hematological parameter LPS do have an important initial clear diagnosis in the differential diagnosis. The initial diagnosis of AP did give clinicians a clear diagnosis and treatment direction.

CRP belongs to nonspecific acute phase reactive protein, the concentration will increase AP idly when tissue damaged or inflammatory stimuli occur in the body (19). Some research has been proved that CRP as a component of the nonspecific immune mechanism can play a role on innate immunity, which mainly take part in the inflammatory defense of organism. Its synthesis is generated by IL- 1 and IL-6 released in bodies, blood level begins to rise after $6 \mathrm{~h}$ abdominal pain attacks, the peak value will be reached after 3 days, the half-life will be reached almost after $19 \mathrm{~h} \mathrm{(20)}$. The Sensitivity and specificity were only $59.8 \%$ and $56.2 \%$ of CRP to diagnose AP. The joint detection with LPS and S-AMY, the specificity could be $96.2 \%$, there were no significant difference between test LPS alone $(\mathrm{P}>0.05)$. This indicates that CRP has low sensitivity and specificity to diagnose $\mathrm{AP}$, which could not diagnose AP alone because of the low accuracy of diagnosis. It has the value to improve the accuracy of AP when it combines with LPS and S-AMY.

S-AMY is more widely used compared to LPS in diagnosis of AP. Generally, these two indicators are 
combined in clinical practice. However, the diagnostic value of LPS was higher than S-AMY and CRP from the outcomes in this study. Hence, it's not of significance for a combined detection of the three indicators. Two hundred and twelve patients were admitted with acute abdominal pain in our center, a total of $107 \mathrm{AP}$ patients was enrolled, there were no patients with only increased S-AMY or CRP but normal LPS. In view of cost evaluation, it cost $50 \mathrm{RMB}$ in combined detection of the three indicators, while it cost $15 \mathrm{RMB}$ in LPS detection. If only LPS was detected in 212 cases of acute abdomen, 7,420 RMB would be saved, which could reduce the waste of resource and save the cost of detection for patients. Compared to imaging examination, detection of LPS is convenient, rapid, cheap, and free from radiation contacting.

According to the results, there was no significant difference between combined detection or detection of LPS alone. LPS do have an important initial clear diagnosis in the differential diagnosis. The initial diagnosis of AP gives clinicians a clear diagnosis and treatment direction. Therefore, LPS could be used as the preferred laboratory examination in diagnosis of AP.

In our further study, patients with a preliminary diagnosis of AP will be staged according to the Atlanta 2012 revised standard for the severity of patients with AP. We will evaluate the relevant hematological parameters (such as IL6, PCT, LDH, etc.) that have now caused heated discussion and widespread attention. Aiming to investigate whether there is higher value of those hematological parameters for the diagnosis of severe AP. The morbidity and mortality of AP are extremely high, early diagnosis, clear etiology, and searching for effective disease evaluation indicators are important to improve AP prognosis and reduce mortality. Although there are many scoring systems and related examinations for the severity and prognosis of $\mathrm{AP}$, it is not clear which is the most effective. The advantages of simple and rapid hematological parameters detection make it continue to be a research hotspot for clinical diagnosis and prognosis evaluation.

\section{Acknowledgments}

Funding: None.

\section{Footnote}

Data Sharing Statement: Available at http://dx.doi. org/10.21037/apm-20-160
Conflicts of Interest: All authors have completed the ICMJE uniform disclosure form (available at http://dx.doi. org/10.21037/apm-20-160). The authors have no conflicts of interest to declare.

Ethical Statement: The authors are accountable for all aspects of the work in ensuring that questions related to the accuracy or integrity of any part of the work are appropriately investigated and resolved. Our study was conducted in accordance with the Declaration of Helsinki (as revised in 2013). The study protocols were approved by the ethics committee of the First Affiliated Hospital of Xinjiang Medical University (approval No. 20140828-02) and received informed consent from all subjects.

Open Access Statement: This is an Open Access article distributed in accordance with the Creative Commons Attribution-NonCommercial-NoDerivs 4.0 International License (CC BY-NC-ND 4.0), which permits the noncommercial replication and distribution of the article with the strict proviso that no changes or edits are made and the original work is properly cited (including links to both the formal publication through the relevant DOI and the license). See: https://creativecommons.org/licenses/by-nc-nd/4.0/.

\section{References}

1. Liu BL, Gong J. Evaluation of biochemical indicators for the diagnosis of acute pancreatitis. Chin J Lab Diagn 2013;17:1850-2.

2. Tadehara M, Okuwaki K, Imaizumi H, et al. Usefulness of serum lipase for early diagnosis of post-endoscopic retrograde cholangiopancreatography pancreatitis. World J Gastrointest Endosc 2019;11:477-85.

3. Wang PX, Shang D. An analysis of the major guidelines for acute pancreatitis at home and abroad. J Hepatopancreatibol Surg 2017;29:1-5.

4. Yokoe M, Takada T, Mayumi T, et al. Japanese guidelines for the management of acute pancreatitis: Japanese Guidelines 2015. J Hepatobiliary Pancreat Sci 2015;22:405-32.

5. Cornett DD, Spier BJ, Eggert AA, et al. The causes and outcome of acute pancreatitis associated with serum lipase. Dig Dis Sci 2011;56:3376-81.

6. Gomez D, Addison A, De Rosa A, et al. Retrospective Study of Patients With Acute Pancreatitis: Is Serum Amylase Still Required? BMJ Open 2012;2:e01471.

7. Cardoso FS, Ricardo LB, Oliveira AM, et al. C-reactive 
protein prognostic accuracy in acute pancreatitis: timing of measurement and cutoff points. Eur J Gastroenterol Hepatol 2013;25:784.

8. Sahin V, Akpinar MB, Sevim E, et al. Preoperative CRP levels is not predictive early renal dysfunction after coronary artery bypass surgery. Int J Clin Exp Med 2015;8:4146-51.

9. Yadav D, Pitchumoni CS. Issues in hyperlipidemic pancreatitis. J Clin Gastroenterol 2003;36:54-62.

10. Cheng P, Yuan YZ. Etiology and classification of acute pancreatitis. Chin J Digestion 2013;33:727-9.

11. Salem TA, Molloy RG, O'Dwyer PJ. Prospective Study on the Role of C-reactive Protein (CRP) in Patients With an Acute Abdomen. Ann R Coll Surg Engl 2007;89:233-7.

12. Basnayake C, Ratnam D. Blood tests for acute pancreatitis. Aust Prescr 2015;38:128-30.

13. Batra HS, Kumar A, Saha TK, et al. Comparative Study of Serum Amylase and Lipase in Acute Pancreatitis Patients. Indian J Clin Biochem 2015;30:230-3.

14. Hameed AM, Lam VWT, Pleass HC. Significant elevations of serum lipase not caused by pancreatitis: a systematic review. HPB (Oxford) 2015;17:99-112.

15. Agarwal N, Pitchumoni CS, Sivaprasad AV. Evaluating tests for acute pancreatitis. Am J Gastroenterol 1990;85:356-66.

16. Hofmeyr S, Meyer C, Warren BL. Serum lipase should be the laboratory test of choice for suspected acute pancreatitis. S Afr J Surg 2014;52:72-5.

17. Committee on the Learning Health Care System in America; Institute of Medicine; Smith M, et al. editors. Best Care at Lower Cost: The Path to Continuously Learning Health Care in America. Washington (DC): National Academies Press (US); 2013 May.

18. Not test for amylase in cases of suspected acute pancreatitis, Instead, test for lipase [Internet]. Choosing Wisely initiative of the American Board of Internal Medicine (ABIM) Foundation, 2016 Sep [cited 2018 Dec 25]. Available online: http://www.choosingwisely.org/ clinician-lists/american-society-clinicalpathology-testingfor-amylase

19. Smith RC, Southwell-Keely J, Chesher D. Should serum pancreatic lipase replace serum amylase as a biomarker of acute pancreatitis? ANZ J Surg 2005;75:399-404.

20. Andersson E, Axelsson J, Eckerwatl G, et al. Tissue factor in predicted severe acute pancreatitis. World J Gastroenterol 2010;16:6128-34.

Cite this article as: Wang L, Qi X, Tian F, Li H, Zhao H, Lv J, Zhou X, Ba D, Song W, Ma X. Diagnostic value of hematological parameters in acute pancreatitis. Ann Palliat Med 2020;9(5):2716-2722. doi: 10.21037/apm-20-160 